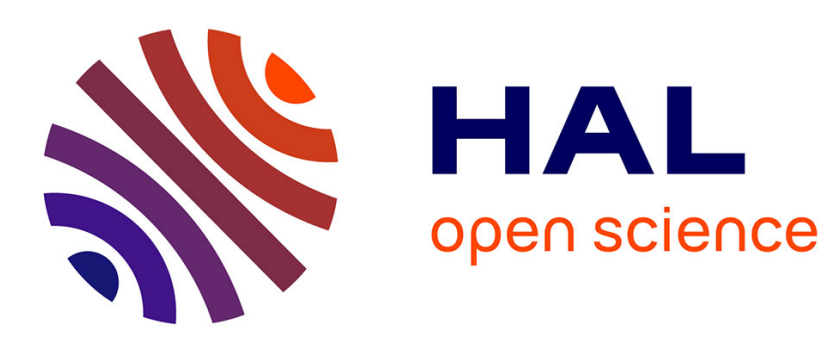

\title{
Improvement of RTK performances using an array of receivers with known geometry
}

Xiao Hu, Paul Thevenon, Christophe Macabiau

\section{To cite this version:}

Xiao Hu, Paul Thevenon, Christophe Macabiau. Improvement of RTK performances using an array of receivers with known geometry. ION ITM 2020, International Technical Meeting, Jan 2020, San Diego, United States. 10.33012/2020.17154 . hal-02549028

\section{HAL Id: hal-02549028 \\ https://hal-enac.archives-ouvertes.fr/hal-02549028}

Submitted on 29 Apr 2020

HAL is a multi-disciplinary open access archive for the deposit and dissemination of scientific research documents, whether they are published or not. The documents may come from teaching and research institutions in France or abroad, or from public or private research centers.
L'archive ouverte pluridisciplinaire HAL, est destinée au dépôt et à la diffusion de documents scientifiques de niveau recherche, publiés ou non, émanant des établissements d'enseignement et de recherche français ou étrangers, des laboratoires publics ou privés. 


\title{
Improvement of RTK performances using an array of receivers with known geometry
}

\author{
Xiao Hu, Paul Thevenon, Christophe Macabiau, ENAC, Université de Toulouse, France
}

\section{BIOGRAPHY}

Xiao Hu is a Ph.D. student within TELECOM laboratory inside the French Civil Aviation University (ENAC), in Toulouse, France. His study is funded by the China Scholarship Council (CSC). He received in 2017 his engineering degree in digital communications and a master's degree in Navigation and Telecommunications from ENAC.

Dr. Paul Thevenon graduated as electronic engineer from Ecole Centrale de Lille in 2004 and obtained in 2007 a research master at ISAE in space telecommunications. In 2010, he obtained a PhD degree in the signal processing laboratory of ENAC in Toulouse, France. From 2010 to 2013, he was employed by CNES, to supervise GNSS research activities. Since the July 2013, he is employed by ENAC as Assistant Professor. His current activities are GNSS signal processing, GNSS integrity monitoring and hybridization of GNSS with other sensors.

Dr. Christophe Macabiau graduated as electronics engineer in 1992 from the ENAC (Ecole Nationale de l'Aviation Civile) in Toulouse, France. Since 1994, he has been working on the application of satellite navigation techniques to civil aviation. He received his Ph.D in 1997 and has been in charge of the signal processing lab of ENAC since 2000, where he also started dealing with navigation techniques for urban navigation. He is currently the head of the TELECOM team of ENAC, that includes research groups on signal processing and navigation, electromagnetics and data communication networks.

\begin{abstract}
Nowadays, a precise position and attitude information is significantly required for specific application scenarios like autonomous driving of vehicles or precise mobile mapping. The GNSS carrier phase measurements appear compulsory to satisfy the sub-meter or even centimeter level need for this kind of requirement. In this paper, we firstly use a method includes an array of receivers with known geometry to enhance the performance of the RTK in different environments. Taking advantages of the attitude information and known geometry of the array of receivers, we are able to improve some internal steps of precise position computation. Different scenarios are conducted including varying the distance between the 2 antennas of the receiver array, the satellite geometry and the amplitude of the noise measurement to validate the influence of the using of an array of receivers. The simulations results show that our multi-receiver RTK system is more robust to noise and degraded satellite geometry, in terms of ambiguity fixing rate, and get a better position accuracy under same conditions when comparing with the single receiver system.
\end{abstract}

\section{INTRODUCTION}

Nowadays, a precise position and attitude information is significantly required for specific application scenarios like autonomous driving of vehicles or precise mobile mapping. However, standard stand-alone Global Navigation Satellite System (GNSS) positioning accuracy is not capable to satisfy the sub-meter or even centimeter level need for this kind of requirement. To achieve a more accurate positioning, the GNSS carrier phase measurements appear mandatory. Nevertheless, they are also less robust and include a so-called integer ambiguity resolution (IAR) that prevents them to be used directly for positioning. In some harsh environment, severe code multipath, frequent cycle slips and losses of lock might also challenge the process of finding the correct IAR thus reduce the accuracy.

To increase confidence and accelerate the IAR process by limiting the search space, restrictions can be established from the use of an array of two or more receivers with prior known and fixed geometry which includes the length of the baseline vectors between the antennas of the receiver array and the orientation of the vectors.

Recently, several studies have focused on the use of array of receivers for attitude determination [1][2] and calibration of magnetic field sensors [3], Daniel et al. [4] developed a method for the recursive estimation of the positioning and attitude problems using GNSS carrier phase observations from an array of receivers, but they calculated the position of each receiver 
separately thus they didn't take advantage of the known geometry. Farhad et al.[5][6] used an adaptive KF for 3-dimensional attitude determination and position estimation of a mobile robot by fusing the information from a system of two RTK GPSs and an IMU, however, they also didn't consider the known geometry of the receiver as a constraint to help improve the performance. Zheng et al. [7] presented a methodology for integrating carrier phase attitude determination and positioning systems by considering one of the receiver pairs in the attitude determination system also used as the rover for the relative positioning system. Nevertheless, their attitude determination and positioning systems remained independent which didn't much ameliorate the success rate of IAR for the RTK positioning. Nandakumaran et al. [8] provided a numerical insight into the role taken by the multi-GNSS integration in delivering high-precision solutions, however, they focused on the PPP solution rather than the RTK processing which requires long convergence time to reach cm-level accuracy. Khodabandeh et al. [9] [10] introduced a concept of array-based between-satellite single difference satellite phase biases determination to accelerate the single-receiver IAR, but they didn't take into account the attitude information of the vehicle which cannot analyze the influence with attitude consideration.

To our knowledge, the use of an array of receivers is not used in order to improve the accuracy of the array position or for some internal steps of precise position computation for RTK processing with attitude determination, such as cycle slip detection or integer ambiguity resolution. Our paper focuses on the improvement of integer ambiguity resolution by using an array of two receivers with known geometry.

\section{MATHEMATICAL MODELS}

In this work, an Extended Kalman Filter (EKF) based position and attitude determination algorithm is developed. The scenario considers a moving vehicle, on which two low-cost receivers were mounted on the roof along the longitudinal axis of the vehicle with a known baseline length, whereas the third one is assumed as static and its position is static and known, the geometry of the model is shown in the figure 1 .

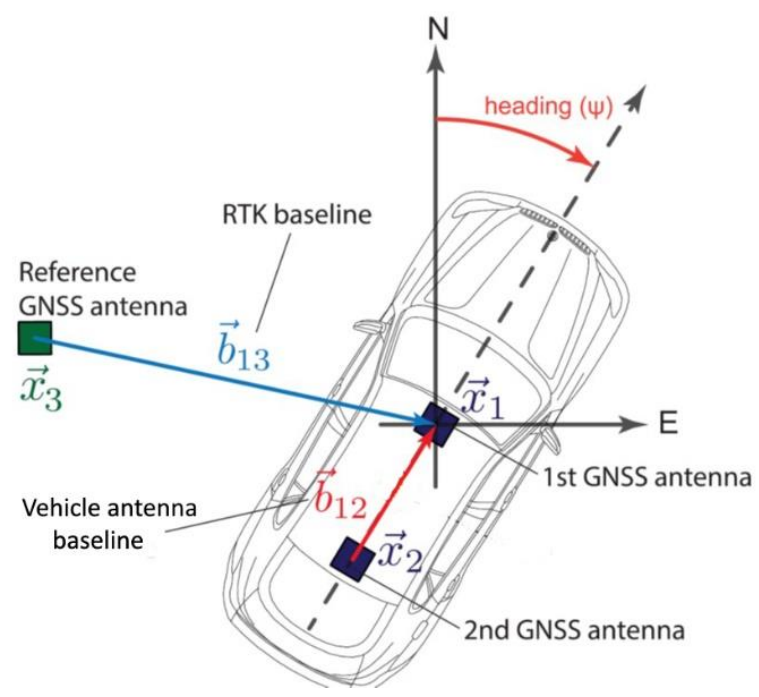

Figure 1: Geometry of the Model [1].

For every time epoch, we estimate both the RTK position and receiver array attitude, the former being defined as a function of the absolute positions $\vec{x}_{1}$ and $\vec{x}_{3}$ :

$$
\vec{b}_{13}\left(\vec{x}_{1}, \vec{x}_{3}\right)=\vec{x}_{1}-\vec{x}_{3}
$$

And the latter being defined as a function of Euler attitude angles with the vehicle antenna baseline length $l=\left\|\vec{b}_{12}\right\|$, the heading $\psi$ and the pitch $\theta$ of the vehicle. The vehicle antenna baseline vector $\vec{b}_{12}$ has the following coordinates in the local navigation (East, North, Up) ENU frame:

$$
\vec{b}_{12}(\psi, \theta)=\left\|\vec{b}_{12}\right\| \cdot\left[\begin{array}{c}
\cos (\theta) \sin (\psi) \\
\cos (\theta) \cos (\psi) \\
\sin (\theta)
\end{array}\right]
$$


It should be noted that the absolute position $\vec{x}_{3}$ of the reference GNSS antenna is static and known and the norm $\left\|\vec{b}_{12}\right\|$ of the attitude baseline is always constant and known a priori, too. Thus, the heading and pitch angle estimates follow from the fixed baseline estimate by simple trigonometric properties as

$$
\begin{gathered}
\hat{\psi}\left(t_{i}\right)=\operatorname{atan}\left(\frac{\left(\vec{b}_{12}\right)_{x}\left(t_{i}\right)}{\left(\vec{b}_{12}\right)_{y}\left(t_{i}\right)}\right) \\
\hat{\theta}\left(t_{i}\right)=\operatorname{atan}\left(\frac{\left(\vec{b}_{12}\right)_{z}\left(t_{i}\right)}{\sqrt{\left(\vec{b}_{12}\right)_{x}^{2}\left(t_{i}\right)+\left(\vec{b}_{12}\right)_{y}^{2}\left(t_{i}\right)}}\right)
\end{gathered}
$$

In the following, the state and the measurement vectors are described along with the state-transition and measurement models.

\section{State transition model}

The state transition or state-space model describes how the states or parameters of the system vary over time based on a specific linear model.

In our KF modeling, the state parameter transition between subsequent epochs is given by:

Where:

$$
\mathbf{x}_{n}=\boldsymbol{\phi}_{n-1} \mathbf{x}_{n-1}+\mathbf{w}_{n}
$$

$\mathbf{x}_{n} \quad$ refers to the state vector at epoch $n$

$\boldsymbol{\phi}_{n-1}$ refers to the transition matrix of epoch $n-1$

$\mathbf{w}_{n} \quad$ refers to the so-called system noise vector at epoch

Together with the process noise vector one can define the process noise covariance matrix as:

$$
\mathbf{Q}_{n}=E\left[\mathbf{w}_{n} \mathbf{w}_{n}^{\mathrm{T}}\right]
$$

This matrix has then the variances of the state parameter's estimates based on the system model.

The estimated parameters are collected inside the state vector. The state vector collects 5 vehicle state parameters and $2 *\left(N_{s a t}-\right.$ 1) satellite state parameters which are: 3D position of GNSS receiver 1 relative to GNSS receiver 3, heading angle of vehicle, pitch angle of vehicle, double-difference integer ambiguity of the GNSS receiver pair 1-3 and the double-difference integer ambiguity of the receiver pair 2-3. which is given by:

$$
\mathbf{x}=\left[\begin{array}{lllll}
\mathbf{b}_{13}^{T} & \theta & \psi & \mathbf{N}_{13}^{T} & \mathbf{N}_{23}^{T}
\end{array}\right]^{T}
$$

where:

$\mathbf{N}_{n p}^{T} \quad$ refers to the vector of the double-difference GPS interger ambiguity between receiver $\mathrm{n}$ and $\mathrm{p}$

\section{Transition model for position and attitude related state parameters}

In our EKF modeling, for the position and attitude related state parameters, we suppose that it's a random walk model, the speed and the angular rate are a zero-mean Gaussian process.

$$
\begin{aligned}
\mathbf{b}_{13, n} & =\mathbf{b}_{13, n-1}+\mathbf{w}_{13, n} \\
\theta_{n} & =\theta_{n-1}+w_{\theta, n} \\
\psi_{n} & =\psi_{n-1}+w_{\psi, n}
\end{aligned}
$$

where:

$\mathbf{w}_{13}$ is a centered Gaussian vector with a covariance matrix $\mathbf{Q}_{13}$ $w_{\theta}$ is a centered Gaussian variable with a standard deviation $\sigma_{\theta}$ $w_{\psi}$ is a centered Gaussian variable with a standard deviation $\sigma_{\psi}$ 


\section{Transition model for satellite-related state parameters}

In case of the satellite-related parameters, they are assumed all as constant over subsequent epochs with a very small noise compared to the position and attitude related state parameters.

where:

$$
\begin{aligned}
& \mathbf{N}_{13, n}=\mathbf{N}_{13, n-1}+\mathbf{w}_{a m b, n} \\
& \mathbf{N}_{23, n}=\mathbf{N}_{23, n-1}+\mathbf{w}_{a m b, n}
\end{aligned}
$$

$\mathbf{w}_{a m b, n}$ is a centered Gaussian vector with a covariance matrx $\mathbf{Q}_{a m b}=\sigma_{a m b}^{2} \mathbf{I}_{N_{s a t}-1}$

$\mathbf{I}_{N}$ is the identity matrix of size $N$

The resulting state transition matrix $\boldsymbol{\phi}$ is then given by a unit matrix and different values of process noise variance are added to complete the model.

$$
\boldsymbol{\Phi}=\left[\begin{array}{ccc}
1 & \cdots & 0 \\
\vdots & \ddots & \vdots \\
0 & \cdots & 1
\end{array}\right]
$$

And the corresponding process noise matrix $\mathbf{Q}$ is given as follows:

$$
\mathbf{Q}=\left[\begin{array}{ccccc}
\mathbf{Q}_{13} & \mathbf{0}_{3 \times 1} & \mathbf{0}_{3 \times 1} & \mathbf{0}_{3 \times N_{s a t}-1} & \mathbf{0}_{3 \times N_{s a t}-1} \\
\mathbf{0}_{1 \times 3} & \sigma_{\theta}^{2} & 0 & \mathbf{0}_{1 \times N_{s a t}-1} & \mathbf{0}_{1 \times N_{s a t}-1} \\
\mathbf{0}_{1 \times 3} & 0 & \sigma_{\psi}^{2} & \mathbf{0}_{1 \times N_{s a t}-1} & \mathbf{0}_{1 \times N_{s a t}-1} \\
\mathbf{0}_{N_{s a t}-1 \times 3} & \mathbf{0}_{N_{s a t}-1 \times 1} & \mathbf{0}_{N_{s a t}-1 \times 1} & \mathbf{Q}_{a m b} & \mathbf{0}_{N_{s a t}-1} \\
\mathbf{0}_{N_{s a t}-1 \times 3} & \mathbf{0}_{N_{s a t}-1 \times 1} & \mathbf{0}_{N_{s a t}-1 \times 1} & \mathbf{0}_{N_{s a t}-1} & \mathbf{Q}_{a m b}
\end{array}\right]
$$

\section{Measurement model}

The measurement model describes how the individual sensor measurements are related to system states. In general, for every epoch $\mathrm{n}$, the measurement vector $\mathbf{z}_{\boldsymbol{n}}$, which contains all measured values, can be described as a function of the state vector $\mathbf{x}_{\boldsymbol{n}}$ as:

$$
\mathbf{z}_{\boldsymbol{n}}=\mathbf{h}_{n}\left(\mathbf{x}_{\boldsymbol{n}}\right)+\mathbf{v}_{n}
$$

with $\mathbf{h}_{n}$ the function that relates one or more states with each measured value and $\mathbf{v}_{n}$ the measurement noise vector, which describes the expected Gaussian noise of every measured value. As we are modeling an extended Kalman filter, $\mathbf{h}_{n}$ is a usually non-linear function.

As for the process noise covariance matrix, the definition of the measurement noise covariance matrix follows as:

$$
\mathbf{R}_{n}=E\left[\mathbf{v}_{n} \mathbf{v}_{n}^{\mathrm{T}}\right]
$$

In our model, the measurement vector comprises the following measured values: Double-difference (DD) code phase measurement vector of receiver 1 , DD code phase measurement vector of receiver 2, DD carrier phase measurement vector of receiver 1 and DD carrier phase measurement vector of receiver 2.

$$
\mathbf{z}_{n}=\left[\begin{array}{lll}
\left(\mathbf{P}_{13}\right)^{T} & \left(\mathbf{P}_{23}\right)^{T} \quad \lambda\left(\boldsymbol{\phi}_{13}\right)^{T} \quad \lambda\left(\boldsymbol{\phi}_{23}\right)^{T}
\end{array}\right]^{T}
$$

In this measurement model, the position of receiver 2 is expressed in terms of the position of receiver 1 and the baseline vector between the 2 receivers of the array, such that it contains the known array baseline length information and the attitude information that we want to estimate. The individual double-difference corrected pseudorange and phase GPS measurement for our short baseline case (less than $1 \mathrm{~km}$ ) can be modeled as:

$$
\begin{gathered}
P_{13}^{k l}=\left(\mathbf{e}^{k l}\right)^{T} \mathbf{b}_{13}+n_{P, 13}^{k l} \\
P_{23}^{k l}=\left(\mathbf{e}^{k l}\right)^{T}\left(\mathbf{b}_{13}-l\left[\begin{array}{c}
\cos \theta \sin \psi \\
\cos \theta \cos \psi \\
\sin \theta
\end{array}\right]\right)+n_{P, 23}^{k l}
\end{gathered}
$$




$$
\begin{aligned}
& \lambda \phi_{13}^{k l}=\left(\mathbf{e}^{k l}\right)^{T} \mathbf{b}_{13}+\lambda N_{13}^{k l}+n_{\phi, 13}^{k l} \\
& \lambda \phi_{23}^{k l}=\left(\mathbf{e}^{k l}\right)^{T} \mathbf{b}_{23}+\lambda N_{23}^{k l}+n_{\phi, 23}^{k l} \\
= & \left(\mathbf{e}^{k l}\right)^{T}\left(\mathbf{b}_{13}-\mathbf{b}_{12}\right)+\lambda N_{23}^{k l}+n_{\phi, 13}^{k l} \\
= & \left(\mathbf{e}^{k l}\right)^{T}\left(\mathbf{b}_{13}-l\left[\begin{array}{c}
\cos \theta \sin \psi \\
\cos \theta \cos \psi \\
\sin \theta
\end{array}\right]\right)+\lambda N_{23}^{k l}+n_{\phi, 13}^{k l}
\end{aligned}
$$

where:

$$
\begin{array}{ll}
P_{n p}^{k l} & \text { refers to the double-difference code phase measurement vector of receiver pair } n p \text { and satellite pair } k l \\
\lambda \phi_{n p}^{k l} & \text { refers to the carrier phase measurement vector of receiver pair } n p \text { and satellite pair } k l \\
\mathbf{e}^{k l} & \text { refers to the difference between the Line of Sight vector of satellite } k \text { and } l \\
N_{n p}^{k l} & \text { refers to the double-difference interger ambiguity of receiver pair } n p \text { and satellite pair } k l \\
n_{P, n p}^{k l}, n_{\phi, n p}^{k l} & \text { refers to the noise measurement of the double difference code and phase measurement respectively }
\end{array}
$$

Additionally, the LOS vector are modelled by using the azimuth $\varphi_{k, r}$ and elevation $\theta_{k, r}$ of the satellites with respect to the corresponding receiver, thanks to the following formula who define the LOS vector between satellite $\mathrm{k}$ and receiver $\mathrm{r}$ in the ENU frame:

$$
\mathbf{e}_{r}^{k}=\left[\begin{array}{c}
\cos \theta_{k, r} \cos \varphi_{k, r} \\
\cos \theta_{k, r} \sin \varphi_{k, r} \\
\sin \theta_{k, r}
\end{array}\right]
$$

To reflect the difference in accuracy between the code measurement and the carrier phase measurement, a fixed scale factor is applied:

where

$$
\sigma_{\text {carrier }}=a * \sigma_{\text {code }}
$$

$\sigma_{\text {code }} \quad$ refers to the standard deviation of code measurement

$\sigma_{\text {carrier }}$ refers to the standard deviation of carrier phase measurement

$a$ refers to the ratio between $\sigma_{\text {code }}$ and $\sigma_{\text {carrier }}$ with a value equals to $1 / 1540$ to account for the much better accuracy of carrier phase measurements

A fixed measurement noise variance between all satellites is defined to complete the measurement model, defining the measurement covariance matrix $\mathbf{R}$. Firstly, the measurement covariance matrix $\mathbf{R}_{\mathrm{SD}}$ for the single difference measurements will have the following shape:

$$
\mathbf{R}_{\text {code }, \mathrm{SD}}=2 * \sigma_{\text {code }}^{2}\left[\begin{array}{cc}
\mathbf{I}_{N_{\text {sat }}} & \mathbf{0}_{\text {Nsat }} \\
\mathbf{0}_{\text {Nsat }} & \mathbf{I}_{N_{\text {sat }}}
\end{array}\right], \quad \mathbf{R}_{\text {carrier,SD }}=2 * a^{2} * \sigma_{\text {code }}^{2}\left[\begin{array}{cc}
\mathbf{I}_{N_{\text {sat }}} & \mathbf{0}_{\text {Nsat }} \\
\mathbf{0}_{N s a t} & \mathbf{I}_{N_{\text {sat }}}
\end{array}\right]
$$

The measurement covariance matrix $\mathbf{R}$ for the double difference measurements can then be deduced using the following formula:

Where

$$
\mathbf{R}=\mathbf{D}\left[\begin{array}{cc}
\mathbf{R}_{\text {code,SD }} & \mathbf{0}_{N_{\text {sat }}} \\
\mathbf{0}_{N_{\text {sat }}} & \mathbf{R}_{\text {carrier,SD }}
\end{array}\right] \mathbf{D}^{T}
$$

$$
\boldsymbol{D}=\left[\begin{array}{ccccc}
-1 & 0 & \cdots & 0 & 1 \\
0 & -1 & \ddots & \vdots & 1 \\
\vdots & \ddots & \ddots & 0 & \vdots \\
0 & \cdots & 0 & -1 & 1
\end{array}\right]
$$

is the single-differencing matrix used for computing the double difference

The relationship between the state and measurement vector is obviously not linear thus we need the EKF to help linearize the non-linear measurement function to the measurement matrix $\mathrm{H}$.

Two alternating steps which are the state prediction step and the state update step are then conducted to complete the algorithm. 


\section{EXTENDED KALMAN FILTER PROCESSING}

The Kalman Filter algorithm is an iterative algorithm that comprises basically two alternating steps: the state prediction step and the state update step. In the first one, the state transition model is applied to predict the behavior of the system in the next epoch basing on a-priori information such as those coming from the movement model. In the second step, the update step, the prediction is confronted with the actual measurements, and a trade-off between the two estimates is chosen as optimal. This optimum is computed basing on the stochastic properties both state transition and measurement model with a Bayesian approach on a MMSE (minimum mean square error) basis.

\section{The prediction step}

In the prediction step, the system model is used to make an estimate on the state variable's value for the subsequent epoch. In this phase, a prediction on the state parameter's values on the subsequent epoch is done only by assuming a linear model, such as a movement model. The equation that describes this prediction uses the section State transition model excluding the state noise component.

where

$$
\hat{\mathbf{x}}_{n}^{-}=\boldsymbol{\Phi}_{n-1} \hat{\mathbf{x}}_{n-1}^{+}
$$

$\hat{\mathbf{x}}_{n-1}^{+}$refers to the estimate of the state vector coming from the last update phase epoch n-1

$\hat{\mathbf{x}}_{n}^{-} \quad$ refers to the estimate of the predicted state vector for the current epoch $\mathrm{n}$

Together with the state vector, there is also another quantity that should be updated: namely the state covariance matrix, which is defined as the expected value of the state vector residuals, which in turn are defined as the difference or error between the real and estimated state vector:

$$
\mathbf{P}=\mathbf{E}\left[(\mathbf{x}-\hat{\mathbf{x}})(\mathbf{x}-\hat{\mathbf{x}})^{\mathrm{T}}\right]
$$

where $\mathbf{x}$ is the true and $\widehat{\mathbf{x}}$ is the estimated state vector. This matrix could be related either to the prediction or update step, in which case the matrix is called a-priori or a-posteriori state covariance matrix.

In the prediction step, the a-priori state covariance matrix $\mathbf{P}_{n}^{-}$is updated as:

$$
\mathbf{P}_{n}^{-}=\mathbf{\Phi}_{n-1} \mathbf{P}_{n-1}^{+} \mathbf{\Phi}_{n-1}^{\mathrm{T}}+\mathbf{Q}_{n-1}
$$

where $\mathbf{P}_{n-1}^{+}$represents the a-posteriori state covariance matrix of the previous state update step.

\section{The update step}

In this step, the measured values are taken into account and "fed back" to the system, the relationship between the state and measurement vector has been mentioned in the Measurement model section:

$$
\mathbf{z}_{n}=\mathbf{h}_{n}\left(\mathbf{x}_{n}\right)+\mathbf{v}_{n}
$$

The non-linear measurement function $\mathbf{h}_{n}\left(\mathbf{x}_{n}\right)$ is linearized to the measurement matrix $\mathbf{H}_{n}$ as:

$$
\mathbf{h}_{n}\left(\mathbf{x}_{n}\right) \approx \mathbf{H}_{n} \mathbf{x}_{n}=\mathbf{h}_{n}\left(\hat{\mathbf{x}}_{n}^{-}\right)+\mathbf{H}_{n}\left(\mathbf{x}_{n}-\hat{\mathbf{x}}_{n}^{-}\right)
$$

which is then used to calculate the Kalman gain matrix $\mathbf{K}_{n}$ and the a-posteriori covariance matrix $\mathbf{P}_{n}^{+}$, as will be seen later in this section. The calculation of the measurement matrix can be then performed as:

In our case

$$
\left.\mathbf{H}_{n}\right|_{\mathbf{x}_{n}=\hat{\mathbf{x}}_{n}^{-}}=\left.\frac{\partial}{\partial \mathbf{x}_{n}} \mathbf{h}_{n}\left(\mathbf{x}_{n}\right)\right|_{\mathbf{x}_{n}=\hat{\mathbf{x}}_{n}^{-}}
$$

$$
\begin{gathered}
\frac{\partial \mathbf{P}_{13}^{k l}}{\partial \mathbf{x}}=\left[\left(\mathbf{e}^{k l}\right)^{T}, 0,0, \mathbf{0}_{1 \times N_{s a t}-1}, \mathbf{0}_{1 \times N_{s a t}-1}\right] \\
\frac{\partial \mathbf{P}_{23}^{k l}}{\partial \mathbf{x}}=\left[\left(\mathbf{e}^{k l}\right)^{T},\left(\mathbf{e}^{k l}\right)^{T} *(-l) *\left[\begin{array}{c}
-\sin \theta \sin \psi \\
-\sin \theta \cos \psi \\
\cos \theta
\end{array}\right],\left(\mathbf{e}^{k l}\right)^{T} *(-l) *\left[\begin{array}{c}
\cos \theta \cos \psi \\
-\cos \theta \sin \psi \\
0
\end{array}\right], \mathbf{0}_{1 \times N_{s a t}-1}, \mathbf{0}_{1 \times N_{s a t}-1}\right]
\end{gathered}
$$




$$
\begin{gathered}
\frac{\partial \boldsymbol{\phi}_{13}^{k l}}{\partial \mathbf{x}}=\left[\left(\mathbf{e}^{k l}\right)^{T}, 0,0, \lambda_{\boldsymbol{k}}, \mathbf{0}_{1 \times N_{s a t}-1}\right] \\
\frac{\partial \boldsymbol{\phi}_{23}^{\mathbf{k l}}}{\partial \mathbf{x}}=\left[\left(\mathbf{e}^{k l}\right)^{T},\left(\mathbf{e}^{k l}\right)^{T} *(-l) *\left[\begin{array}{c}
-\sin \theta \sin \psi \\
-\sin \theta \cos \psi \\
\cos \theta
\end{array}\right],\left(\mathbf{e}^{k l}\right)^{T} *(-l) *\left[\begin{array}{c}
\cos \theta \cos \psi \\
-\cos \theta \sin \psi \\
0
\end{array}\right], \mathbf{0}_{1 \times N_{s a t}-1}, \lambda_{\boldsymbol{k}}\right]
\end{gathered}
$$

where $\lambda_{\boldsymbol{k}}$ is a vector of $N_{s a t}-1$ zero, except for one value which is equal to $\lambda_{G P S}$ in the k-th position.

So, the corresponding Matrix $\mathbf{H}$ can be defined as follows:

$$
\mathbf{H}=\left[\begin{array}{ccccccccccc}
e_{e}^{1} & e_{n}^{1} & e_{u}^{1} & 0 & 0 & 0 & \ldots & 0 & 0 & \ldots & 0 \\
\vdots & \vdots & \vdots & \vdots & \vdots & \vdots & \ddots & \vdots & \vdots & \ddots & \vdots \\
e_{e}^{\text {Nsat }-1} & e_{n}^{\text {Nsat }-1} & e_{u}^{\text {Nsat }-1} & 0 & 0 & 0 & \ldots & 0 & 0 & \ldots & 0 \\
e_{e}^{1} & e_{n}^{1} & e_{u}^{1} & h_{\theta} & h_{\psi} & 0 & \ldots & 0 & 0 & \ldots & 0 \\
\vdots & \vdots & \vdots & \vdots & \vdots & \vdots & \ddots & \vdots & \vdots & \ddots & \vdots \\
e_{e}^{\text {Nsat }-1} & e_{n}^{\text {Nsat }-1} & e_{u}^{\text {Nsat }-1} & h_{\theta} & h_{\psi} & 0 & \ldots & 0 & 0 & \ldots & 0 \\
e_{e}^{1} & e_{n}^{1} & e_{u}^{1} & 0 & 0 & \lambda^{G P S} & \ldots & 0 & 0 & \ldots & 0 \\
\vdots & \vdots & \vdots & \vdots & \vdots & 0 & \ddots & 0 & 0 & \ddots & 0 \\
e_{e}^{\text {Nat }-1} & e_{n}^{\text {Nat }}-1 & e_{u}^{\text {Nat }}-1 & 0 & 0 & 0 & \ldots & \lambda^{G P S} & 0 & \ldots & 0 \\
e_{e}^{1} & e_{n}^{1} & e_{u}^{1} & h_{\theta} & h_{\psi} & 0 & \ldots & 0 & \lambda^{G P S} & 0 & 0 \\
\vdots & \vdots & \vdots & \vdots & \vdots & 0 & \ddots & 0 & \vdots & \ddots & \vdots \\
e_{e}^{\text {Nsat }-1} & e_{n}^{\text {Nsat }-1} & e_{u}^{\text {Nsat }-1} & h_{\theta} & h_{\psi} & 0 & \ldots & 0 & 0 & 0 & \lambda^{G P S}
\end{array}\right]
$$

with $h_{\theta}=\left(e^{k l}\right)^{T} *(-l) *\left[\begin{array}{c}-\sin \theta \sin \varphi \\ -\sin \theta \cos \varphi \\ \cos \theta\end{array}\right]$ and $h_{\psi}=\left(e^{k l}\right)^{T} *(-l) *\left[\begin{array}{c}\cos \theta \cos \varphi \\ -\cos \theta \sin \varphi \\ 0\end{array}\right]$ respectively.

Note that the values of $\mathbf{H}$ are computed around the predicted position $\hat{\mathbf{x}}_{n}^{-}$

The update of the state vector is performed as:

$$
\widehat{\mathbf{x}}_{n}^{+}=\widehat{\mathbf{x}}_{n}^{-}+\mathbf{K}_{n}\left(\mathbf{z}_{n}-\mathbf{h}_{n}\left(\widehat{\mathbf{x}}_{n}^{-}\right)\right)
$$

where $\mathbf{K}_{n}$ stands for the Kalman gain matrix and the term $\mathbf{z}_{n}-\mathbf{h}_{n}\left(\widehat{\mathbf{x}}_{n}^{-}\right)$is defined as measurement innovation. This discrepancy between what the sensors are telling and what the linear model is pointing out is weighted by the Kalman gain matrix $\mathbf{K}_{n}$ and then finally added to the just calculated predicted state vector $\widehat{\mathbf{x}}_{n}^{-}$coming from the last prediction phase.

The a-posteriori state covariance matrix is updated as follows:

$$
\mathbf{P}_{n}^{+}=\left(\mathbf{I}-\mathbf{K}_{n} \mathbf{H}_{n}\right) \mathbf{P}_{n}^{-}
$$

with $\mathbf{I}$ being the identity matrix, and $\mathbf{K}_{n}$ the Kalman gain matrix, which is defined as:

$$
\mathbf{K}_{n}=\mathbf{P}_{n}^{-} \mathbf{H}_{n}^{T}\left(\mathbf{H}_{n} \mathbf{P}_{n}^{-} \mathbf{H}_{n}^{T}+\mathbf{R}_{n}\right)^{-1}
$$

The updated state vector $\widehat{\mathbf{x}}_{n}^{+}$contains now state estimates that are optimal, considering both the new information provided by the measurements and the prediction of the linear model. This procedure should be iterated as long as new measurement data is available.

\section{RTK PROCESSING}

In the previous process, we obtain a float estimation of the double difference integer ambiguity. The accuracy of the position state estimate is further improved by fixing the DD ambiguities to integer number by using the well-known LAMBDA [11] [12] algorithm. This algorithm has shown its superiority in terms of both performance and processing time when compared to other algorithm in [13]. 
One selects the integer candidates based on the sum of squared errors to get a fixed solution. The candidate with the lowest error norm is chosen once the ratio of the Maximum A Posteriori error norm between the second-best candidate and the best candidate is bigger than a threshold. It is a pre-defined threshold or the critical value that the squared norm of ambiguity residuals of the best and second best candidates should overpass to validate the integer estimation. In our paper, an empirical fixed value 3 is taken as in [14]

Once the IAR process is declared successful, a new position is computed using the DD carrier phase measurements corrected by the validated DD integer ambiguities. This final position is the fixed solution.

\section{SIMULATION OF RTK PERFORMANCES USING AN ARRAY OF RECEIVERS}

In this section, the proposed precise position and attitude determination algorithm is verified with simulated measurements. The reason why we use the simulated measurements is that we want to see the advantage of using an array of receivers with known geometry when varying the length of the baseline, the noise measurement and the satellite geometry when compared to the traditional single receiver system. To validate the interest of the use of an array of receivers, several scenarios are conducted, in all the scenarios, we conduct the analysis by comparing between single receiver RTK and multi-receiver RTK in terms of fixing rate and position accuracy under same conditions.

The performance criteria shown in the presented tables are obtained by computing the mean, standard deviation and $95 \%$ bound of indicators, based on the running of 100 Monte-Carlo simulation, where the noise and initial states are drawn randomly.

To get enough change of the attitude of the vehicle during the simulation in order to observe the algorithm performance for different heading and pitch, the vehicle is assumed to make a uniform circular motion around the fixed reference receiver 3 with a constant radius $(\mathrm{R}=100 \mathrm{~m})$ in all the scenarios. The relative horizontal positions of the dual-receiver system and the single receiver system in time are shown in the left side and middle of figure 2 respectively.

In order to make the pitch angle and the relative altitude between receiver 1 and 2 observable, the ground is considered to be undulated thus we also add a small altitude and pitch variation as shown in the right side of figure 2 . The speed of the vehicle is $10 \mathrm{~m} / \mathrm{s}$, the duration of the simulated trajectory is $1000 \mathrm{~s}$ and the sampling rate is $1 \mathrm{~Hz}$

In our simulator, the satellites are simulated with a fixed position (Figure 3). We consider 7 satellites with a fairly good geometry, i.e. well-spaced in azimuth and elevation. This would correspond to a typical case of a single constellation in open sky environment.

Finally, the DD carrier ambiguities are taken as fixed values separated by 10 cycles. This means that the DD ambiguities go from 10 to 120 .
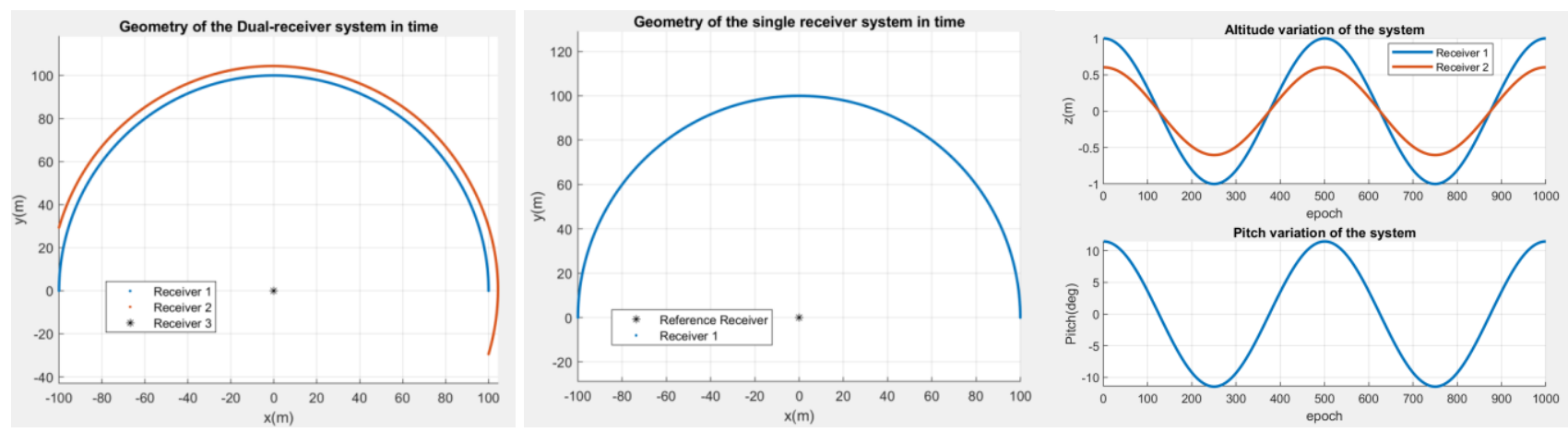

Figure 2: Relative position between the moving receivers in the vehicle and the reference receiver 


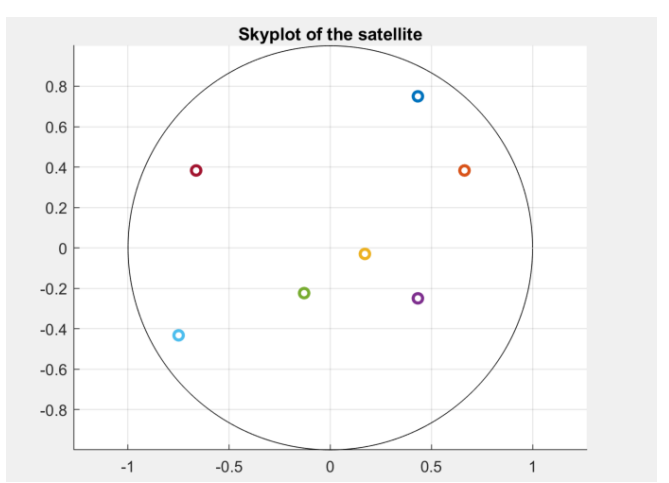

Figure 3: considered satellite geometry

\section{Scenario 1: Impact of the length of the receiver array baseline}

A precise attitude information is essential for autonomous driving of any vehicle, by using an array of receivers, we can additionally get the attitude information of the vehicle. However, this attitude information is calculated based on the known geometry of the multi-receiver system in which the length of the baseline between the two receivers of the array plays an important role. That is why in the first scenario, we want to analyze the influence of system when the length of the baseline changes. The geometry of the array of receivers is then not fixed in this situation, by varying the receiver baseline while keeping the same noise measurement $\left(\sigma_{\text {code }}=1 \mathrm{~m}\right.$ ) and a favorable satellite geometry (all 7 satellites in Figure 3), we confirmed the effect of the length of the array baseline on the performance of the RTK and attitude determination.

Typical results of the state estimations including the estimation of the integer ambiguity resolution (last row) by using LAMBDA are given in figure 4. The top 3 rows show the estimation of the East, North and Up position coordinate respectively whereas the $4^{\text {th }}$ and $5^{\text {th }}$ rows show the estimation of the pitch and heading angles of the vehicle. Figure 5 gives the error comparison between the float and fixed solution. We can see from the result that some convergence period is present on all states. Once they converge, the error between the estimated result and the true value is extremely small.

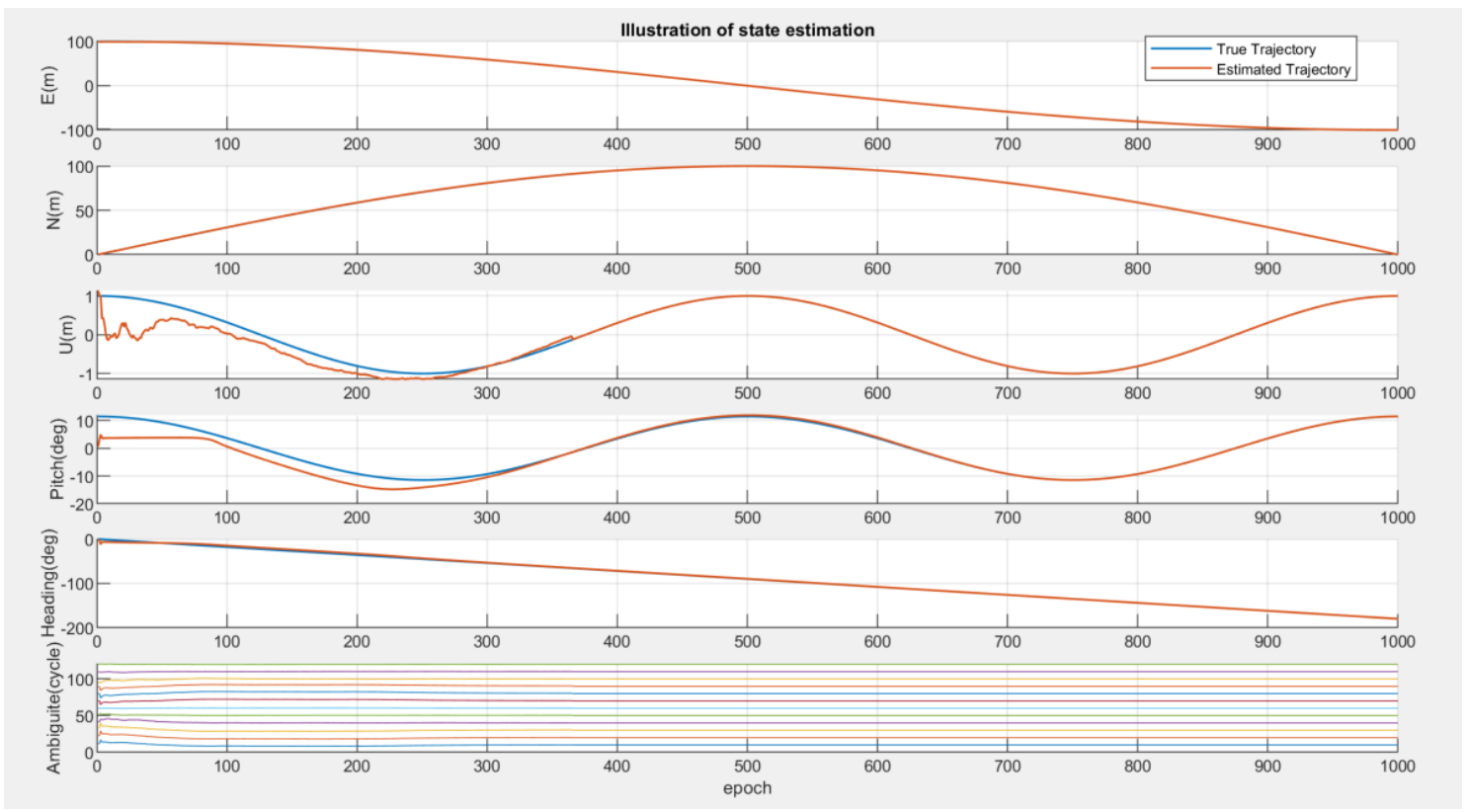

Figure 4: Illustration of state estimation

In Figure 5, the fixed solution is defined by two parts, for every epoch, if the IAR process is declared successful, a new position is computed using the DD carrier phase measurements corrected by the validated DD integer ambiguities. This position then is used to update the fixed solution. If the the IAR process is not declared successful, we keep the float solution. From the figure we can see the system fixes the IAR in the $366^{\text {th }}$ epoch and once the IAR is fixed, the positioning results become much more accurate compared to the float solution. However, in terms of the heading and the pitch, the precision is not much improved in the fixed solution because it's precison is already good enough in the float solution. 


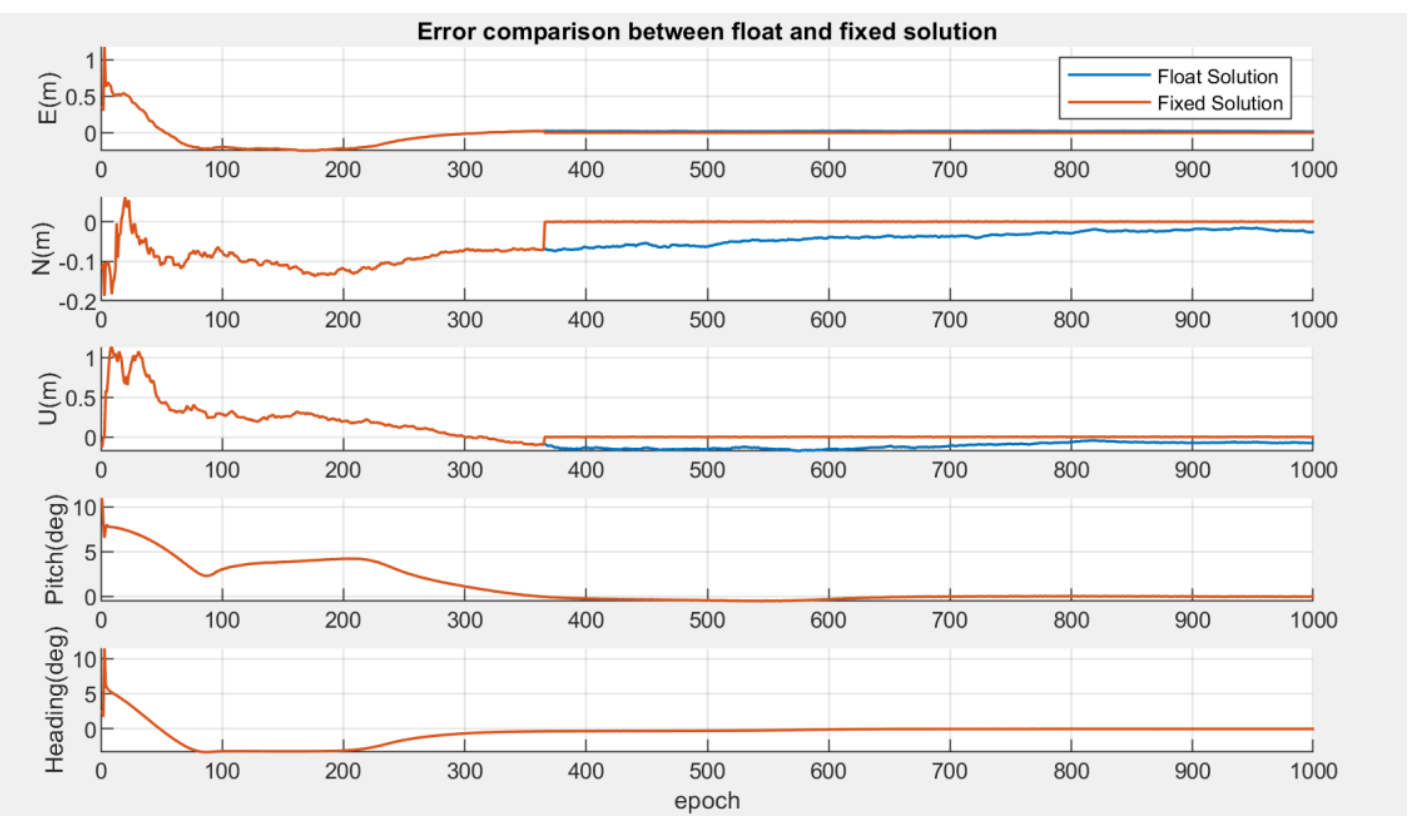

Figure 5: Illustration of float vs fixed solution

Results are compared in terms of fixed rate, 3D position error statistics (mean, standard deviation and 95\% bound) and array attitude error statistics. The numbers have been computed by running 100 Monte-Carlo simulations with different random realization of noise and initialization values and taking the average of the investigated indicators.

The analysis given in Table 1 shows that the dual receiver array system provides better performance than the single receiver RTK solution. Actually, the fixed rate reaches a threshold value of $94.69 \%$ for every multi-receiver solution, which corresponds to the maximum fixed rate after the brief convergence period. We can also see that, as expected, the attitude accuracy improves as the array baseline increases. However, given the favorable assumptions, a baseline of $2 \mathrm{~m}$ is acceptable and would be practical to be installed on real vehicle.

Table 1 - Performance comparison for different array baseline, $\sigma_{\text {code }}=1 \mathrm{~m}, N_{\text {sat }}=7$

\begin{tabular}{|c|c|c|c|c|c|}
\hline $\begin{array}{c}\text { Variation of } \\
\text { scenario } 1\end{array}$ & Performance indicator & Unit & Mean & $\begin{array}{l}\text { Standard } \\
\text { deviation }\end{array}$ & $95 \%$ bound \\
\hline \multirow{4}{*}{ Single receiver } & 3D position error & $\mathrm{m}$ & 0.1725 & 0.2591 & 0.0272 \\
\hline & Absolute heading error & deg & $\mathrm{N} / \mathrm{A}$ & N/A & $\mathrm{N} / \mathrm{A}$ \\
\hline & Absolute pitch error & deg & $\mathrm{N} / \mathrm{A}$ & $\mathrm{N} / \mathrm{A}$ & $\mathrm{N} / \mathrm{A}$ \\
\hline & Fixed rate & $\%$ & 89.35 & 16.08 & 51.13 \\
\hline \multirow{4}{*}{ Dual receiver } & 3D position error & $\mathrm{m}$ & 0.1410 & 0.2089 & 0.0022 \\
\hline & Absolute heading error & deg & 3.9229 & 4.9453 & 0.0528 \\
\hline & Absolute pitch error & deg & 0.9250 & 1.8582 & 0.0495 \\
\hline & Fixed rate & $\%$ & 94.69 & 12.37 & 67.23 \\
\hline \multirow{4}{*}{ Dual receiver } & 3D position error & $\mathrm{m}$ & 0.1185 & 0.1940 & 0.0018 \\
\hline & Absolute heading error & deg & 1.8666 & 2.8054 & 0.0071 \\
\hline & Absolute pitch error & deg & 0.7824 & 1.2755 & 0.0455 \\
\hline & Fixed rate & $\%$ & 94.69 & 12.37 & 67.23 \\
\hline \multirow{4}{*}{ Dual receiver } & 3D position error & $\mathrm{m}$ & 0.0723 & 0.1546 & 0.0019 \\
\hline & Absolute heading error & deg & 1.5194 & 2.3028 & 0.0044 \\
\hline & Absolute pitch error & deg & 0.4820 & 1.3681 & 0.0083 \\
\hline & Fixed rate & $\%$ & 94.69 & 12.37 & 67.23 \\
\hline
\end{tabular}


To be more straightforward, the comparisons of several key parameters between the dual receiver array system and the single receiver system includes the 3D position error, the fixed rate, the heading error and the pitch error are shown in the figure 6.
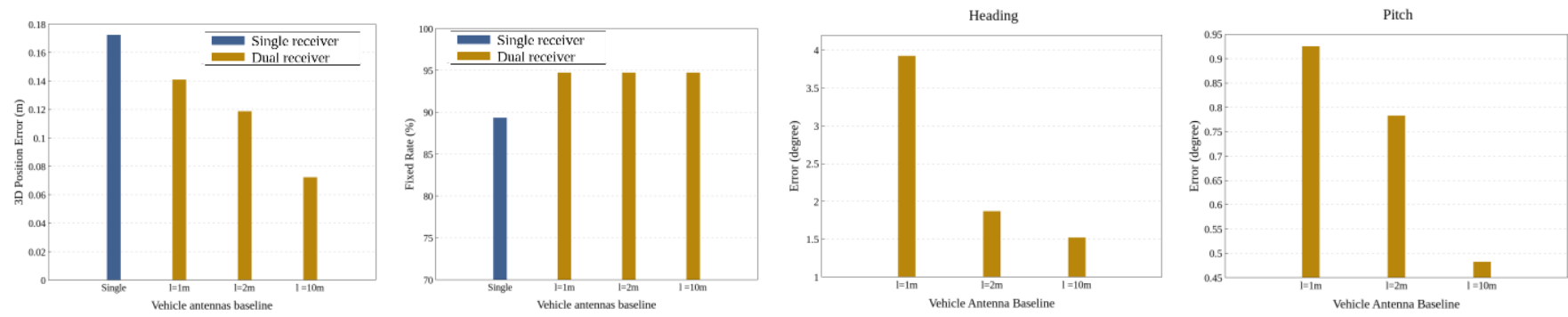

Figure 6: The comparisons of several key parameters in function of length of array baseline

\section{Scenario 2: Noise measurement effect Analyses}

In the second scenario, the geometry of the array of receivers $(l=2 \mathrm{~m})$ and the satellite geometry $\left(N_{s a t}=7\right)$ are fixed, while varying noise measurement $\sigma_{\text {code }}$, we observe the robustness against noise of the multi-receiver RTK system. We recall that the phase measurement noise standard deviation is scaled by a factor $1 / 1540$, to account for the ratio between the wavelength and the GPS L1C/A code chip length.

The analysis of Table 2 shows that the use of the receiver array improves the fixed rate for all considered $\sigma_{\text {code }}$ values, thus demonstrating the interest of such approach.

Table 2 - Performance comparison for different measurement noise, $l=2 \mathrm{~m}, N_{\text {sat }}=7$

\begin{tabular}{|c|c|c|c|c|c|}
\hline $\begin{array}{c}\text { Variation of } \\
\text { scenario } 2\end{array}$ & Performance indicator & Unit & Mean & $\begin{array}{l}\text { Standard } \\
\text { deviation }\end{array}$ & $95 \%$ bound \\
\hline \multirow{2}{*}{ Single receiver } & 3D position error & $\mathrm{m}$ & 0.1725 & 0.2591 & 0.0272 \\
\hline & Absolute heading error & deg & N/A & $\mathrm{N} / \mathrm{A}$ & N/A \\
\hline \multirow{2}{*}{$\sigma_{\text {code }}=1 \mathrm{~m}$} & Absolute pitch error & deg & N/A & N/A & $\mathrm{N} / \mathrm{A}$ \\
\hline & Fixed rate & $\%$ & 89.35 & 16.08 & 51.13 \\
\hline \multirow{2}{*}{ Dual receiver } & 3D position error & $\mathrm{m}$ & 0.1185 & 0.1940 & 0.0019 \\
\hline & Absolute heading error & deg & 1.8666 & 2.8054 & 0.0071 \\
\hline \multirow{2}{*}{$\sigma_{\text {code }}=1 \mathrm{~m}$} & Absolute pitch error & deg & 1.0524 & 1.2755 & 0.0455 \\
\hline & Fixed rate & $\%$ & 94.69 & 12.37 & 67.23 \\
\hline \multirow[b]{2}{*}{ Single receiver } & 3D position error & $\bar{m}$ & 0.1815 & 0.2672 & 0.0076 \\
\hline & Absolute heading error & deg & N/A & N/A & $\mathrm{N} / \mathrm{A}$ \\
\hline \multirow{2}{*}{$\sigma_{\text {code }}=2 \mathrm{~m}$} & Absolute pitch error & deg & $\mathrm{N} / \mathrm{A}$ & $\mathrm{N} / \mathrm{A}$ & $\mathrm{N} / \mathrm{A}$ \\
\hline & Fixed rate & $\%$ & 66.25 & 18.04 & 28.82 \\
\hline \multirow{2}{*}{ Dual receiver } & 3D position error & $\mathrm{m}$ & 0.1338 & 0.2095 & 0.0119 \\
\hline & Absolute heading error & deg & 2.2210 & 3.2912 & 0.1163 \\
\hline \multirow{2}{*}{$\sigma_{\text {code }}=2 \mathrm{~m}$} & Absolute pitch error & deg & 1.1442 & 1.9707 & 0.0213 \\
\hline & Fixed rate & $\%$ & 79.66 & 18.48 & 38.56 \\
\hline \multirow{2}{*}{ Single receiver } & 3D position error & $m$ & 0.3456 & 0.2886 & 0.1597 \\
\hline & Absolute heading error & deg & $\mathrm{N} / \mathrm{A}$ & $\mathrm{N} / \mathrm{A}$ & $\mathrm{N} / \mathrm{A}$ \\
\hline \multirow{2}{*}{$\sigma_{\text {code }}=10 \mathrm{~m}$} & Absolute pitch error & deg & $\mathrm{N} / \mathrm{A}$ & $\mathrm{N} / \mathrm{A}$ & $\mathrm{N} / \mathrm{A}$ \\
\hline & Fixed rate & $\%$ & 19.44 & 4.47 & 11.94 \\
\hline \multirow{2}{*}{ Dual receiver } & 3D position error & $\mathrm{m}$ & 0.1768 & 0.2584 & 0.0346 \\
\hline & Absolute heading error & deg & 3.6595 & 4.7813 & 0.0577 \\
\hline \multirow{2}{*}{$\sigma_{\text {code }}=10 \mathrm{~m}$} & Absolute pitch error & deg & 3.8268 & 7.3318 & 0.2472 \\
\hline & Fixed rate & $\%$ & 24.98 & 6.58 & 13.39 \\
\hline
\end{tabular}


As in the first scenario, the figure 7 gives the comparisons of several key parameters between the dual receiver array system and the single receiver system includes the $3 \mathrm{D}$ position error, the fixed rate, the heading error and the pitch error.
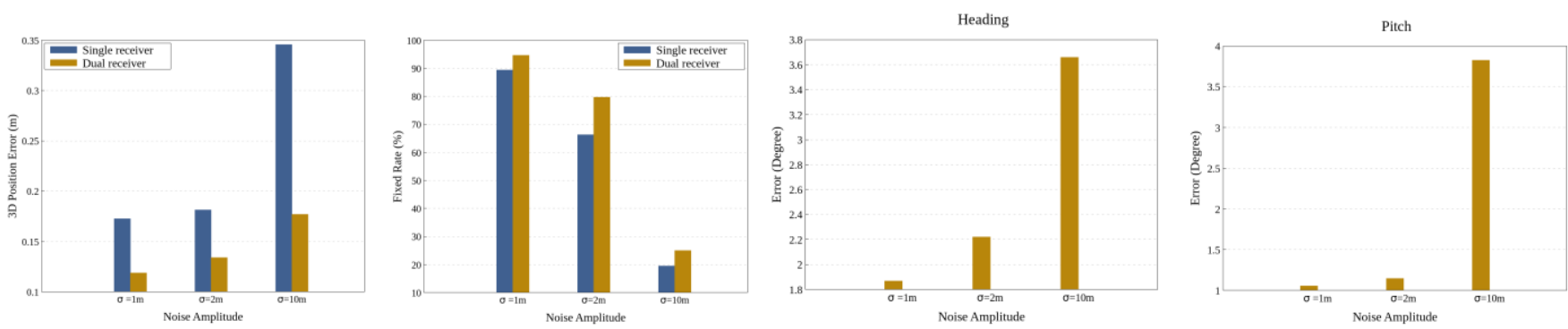

Figure 7: The comparisons of several key parameters in function of measurement noise

\section{Scenario 3: Geometry of satellite effect Analyses}

In the third scenario, the geometry of the array $(l=2 \mathrm{~m})$ and the noise measurement $\left(\sigma_{\text {code }}=1 \mathrm{~m}\right)$ are fixed and by degrading the geometry of the satellite (by removing one satellite at a time), we observe the robustness against degraded satellite geometry of multi-receiver RTK systems. The different scenarios are shown in figure 8
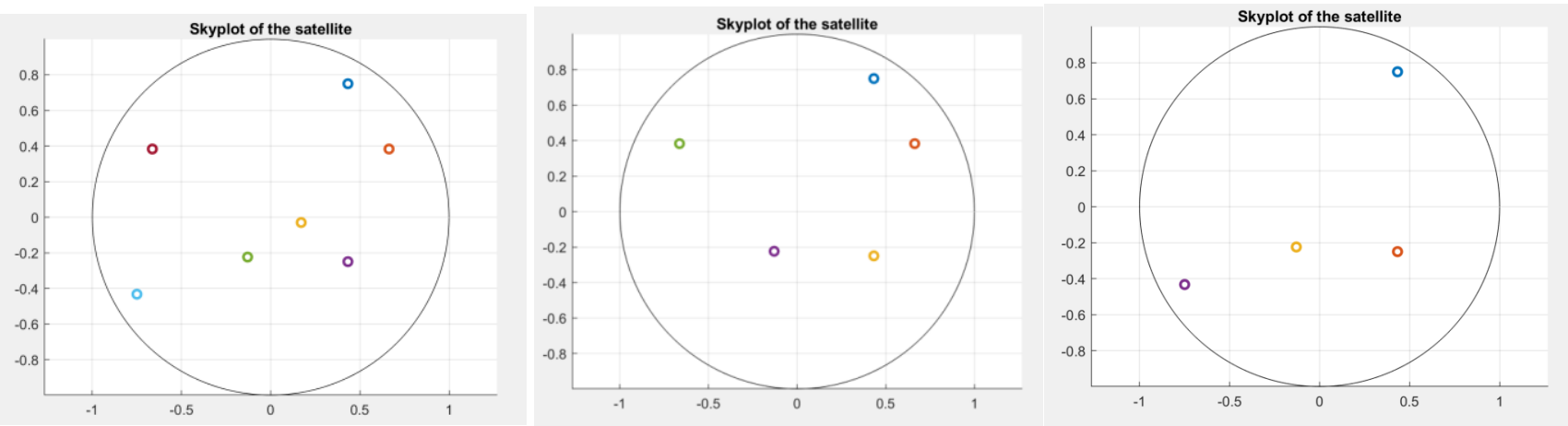

Figure 8: considered satellite geometry with $N_{\text {sat }}=7$ (left), $N_{\text {sat }}=5$ (middle) and $N_{\text {sat }}=4$ (right)

The analysis of Table 3 shows that the use of the receiver array improves the fixed rate for all considered satellites geometry, besides, the dual receiver array provides always better performance than the single receiver RTK solution under the same satellites geometry situation which confirm the interest of our approach.

Table 3 - Performance comparison for different satellite geometry, $l=2 \mathrm{~m}, \sigma_{\text {code }}=1 \mathrm{~m}$

\begin{tabular}{|c|c|c|c|c|c|}
\hline $\begin{array}{c}\text { Variation of } \\
\text { scenario 3 }\end{array}$ & Performance indicator & Unit & Mean & $\begin{array}{c}\text { Standard } \\
\text { deviation }\end{array}$ & 95\% bound \\
\hline \multirow{2}{*}{ Single receiver } & 3D position error & $\mathrm{m}$ & 0.1725 & 0.2591 & 0.0272 \\
\cline { 2 - 6 } & Absolute heading error & $\mathrm{deg}$ & $\mathrm{N} / \mathrm{A}$ & $\mathrm{N} / \mathrm{A}$ & $\mathrm{N} / \mathrm{A}$ \\
\cline { 2 - 6 }$N_{\text {sat }}=7$ & Absolute pitch error & $\mathrm{deg}$ & $\mathrm{N} / \mathrm{A}$ & $\mathrm{N} / \mathrm{A}$ & $\mathrm{N} / \mathrm{A}$ \\
\cline { 2 - 6 } & Fixed rate & $\%$ & 89.35 & 16.08 & 51.13 \\
\hline \multirow{2}{*}{ Dual receiver } & 3D position error & $\mathrm{m}$ & 0.1185 & 0.1940 & 0.0018 \\
\cline { 2 - 6 }$N_{\text {sat }}=7$ & Absolute heading error & $\mathrm{deg}$ & 1.8666 & 2.8054 & 0.0071 \\
\cline { 2 - 6 } & Absolute pitch error & $\mathrm{deg}$ & 1.0524 & 1.2755 & 0.0455 \\
\hline \multirow{2}{*}{ Single receiver } & Fixed rate & $\%$ & 94.69 & 12.37 & 67.23 \\
\cline { 2 - 6 } & 3D position error & $\mathrm{m}$ & 0.5111 & 0.4649 & 0.3116 \\
\cline { 2 - 6 }$N_{\text {sat }}=5$ & Absolute heading error & deg & N/A & N/A & N/A \\
\cline { 2 - 6 } & Absolute pitch error & deg & N/A & N/A & N/A \\
\hline
\end{tabular}




\begin{tabular}{|c|c|c|c|c|c|}
\hline \multirow{3}{*}{ Dual receiver } & 3D position error & $\mathrm{m}$ & 0.4194 & 0.2276 & 0.0322 \\
\cline { 2 - 6 }$N_{\text {sat }}=5$ & Absolute heading error & $\mathrm{deg}$ & 2.1331 & 3.8637 & 0.0871 \\
\cline { 2 - 6 } & Absolute pitch error & $\mathrm{deg}$ & 2.3642 & 2.4387 & 0.0455 \\
\cline { 2 - 6 } & Fixed rate & $\%$ & 84.40 & 13.60 & 66.08 \\
\hline \multirow{3}{*}{ Single receiver } & 3D position error & $\mathrm{m}$ & 0.6264 & 0.2324 & 0.4812 \\
\cline { 2 - 6 } & Absolute heading error & $\mathrm{deg}$ & $\mathrm{N} / \mathrm{A}$ & $\mathrm{N} / \mathrm{A}$ & $\mathrm{N} / \mathrm{A}$ \\
\cline { 2 - 6 }$N_{\text {sat }}=4$ & Absolute pitch error & $\mathrm{deg}$ & $\mathrm{N} / \mathrm{A}$ & $\mathrm{N} / \mathrm{A}$ & $\mathrm{N} / \mathrm{A}$ \\
\cline { 2 - 6 } & Fixed rate & $\%$ & 0.82 & 0.49 & 0.11 \\
\hline \multirow{2}{*}{ Dual receiver } & 3D position error & $\mathrm{m}$ & 0.5432 & 0.3618 & 0.0758 \\
\cline { 2 - 6 } & Absolute heading error & $\mathrm{deg}$ & 3.0569 & 3.3308 & 1.7031 \\
\cline { 2 - 6 }$N_{\text {sat }}=4$ & Absolute pitch error & $\mathrm{deg}$ & 3.7220 & 3.0152 & 2.8678 \\
\cline { 2 - 6 } & Fixed rate & $\%$ & 1.86 & 1.20 & 0.18 \\
\hline
\end{tabular}

To show the results more straightforward, the figure 9 gives the 3D position error, the fixed rate, the heading error and the pitch error comparisons between the dual receiver array system and the single receiver system.
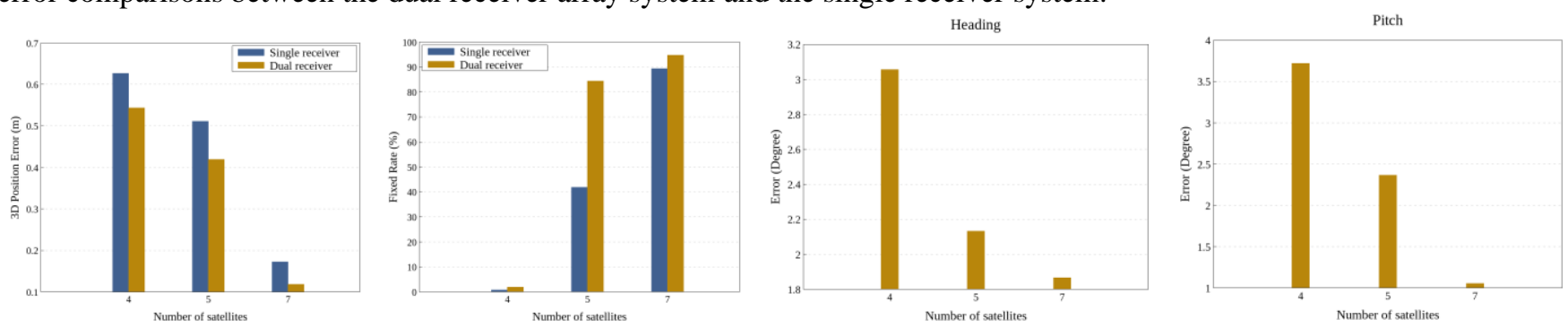

Figure 9: The comparisons of several key parameters in function of satellite geometry

\section{CONCLUSION}

In this paper, we present a method includes an array of receivers with known geometry to enhance the performance of the RTK in different environments. Taking advantages of the attitude information and known geometry of the array of receivers, we are able to improve some internal steps of precise position computation. We demonstrate through simulations results that our multireceiver RTK system is more robust to noise and degraded satellite geometry, in terms of ambiguity fixing rate, and get a better position accuracy under same conditions when comparing with the single receiver system.

Based on this proof-of-concept, several future works are possible. The current simulator could be improved in order to consider more realistic conditions, such as an elevation-dependent measurement noise, moving satellites from real almanac or multiconstellation observations. Arrays with more than 2 receivers could be assessed, as well as the consideration of several receivers connected to the same antenna (zero vehicle antenna baseline configuration).

We also plan to investigate the interest of such array for cycle slip detection and repair, and the mitigation of NLOS conditions permitted by the spatial diversity of the array.

Finally, the concept could be tested against real conditions by setting-up an experiment on a test vehicle.

\section{ACKNOWLEDGEMENTS}

This work has been supported by China Scholarship Council (CSC). It is the Chinese Ministry of Education's non-profit organization that provides support for international academic exchange with China.

\section{REFERENCES}

[1] M. Iafrancesco, "GPS/INS Tightly coupled position and attitude determination with low-cost sensors Master Thesis," p. 69.

[2] P. Henkel and C. Gunther, “Attitude determination with low-cost GPS/ INS,” p. 9.

[3] P. Henkel, P. Berthold, and J. J. Kiam, "Calibration of magnetic field sensors with two mass-market GNSS receivers," in 2014 11th Workshop on Positioning, Navigation and Communication (WPNC), Dresden, Germany, 2014, pp. 1-5. 
[4] D. Medina, A. Heselbarth, R. Buscher, R. Ziebold, and J. Garcia, "On the Kalman filtering formulation for RTK joint positioning and attitude quaternion determination," in 2018 IEEE/ION Position, Location and Navigation Symposium (PLANS), Monterey, CA, 2018, pp. 597-604.

[5] F. Aghili and A. Salerno, "Attitude determination and localization of mobile robots using two RTK GPSs and IMU," in 2009 IEEE/RSJ International Conference on Intelligent Robots and Systems, St. Louis, MO, USA, 2009, pp. $2045-2052$.

[6] F. Aghili and A. Salerno, "3-D Localization of mobile robots and its observability analysis using a pair of RTK GPSs and an IMU," in 2010 IEEE/ASME International Conference on Advanced Intelligent Mechatronics, Montreal, QC, Canada, 2010, pp. 303-310.

[7] G. Zheng and D. Gebre-Egziabher, "Enhancing Ambiguity Resolution Performance Using Attitude Determination Constraints," in 22nd International Technical Meeting of The Satellite Division of the Institute of Navigation, Savannah, Georgia, 2009.

[8] X. Li, M. Ge, M. Fritsche, Y. Liu, J. Wickert, and H. Schuh, "Multi-GNSS Real-time Precise Point Positioning: GPS, GLONASS, BeiDou, and Galileo," p. 22.

[9] A. Khodabandeh and P. J. G. Teunissen, "Single-Epoch GNSS Array Integrity: An Analytical Study," in VIII HotineMarussi Symposium on Mathematical Geodesy, vol. 142, N. Sneeuw, P. Novák, M. Crespi, and F. Sansò, Eds. Cham: Springer International Publishing, 2015, pp. 263-272.

[10] A. Khodabandeh and P. J. G. Teunissen, "Array-based satellite phase bias sensing: theory and GPS/BeiDou/QZSS results," Meas. Sci. Technol., vol. 25, no. 9, p. 095801, Sep. 2014.

[11] P. J. G. Teunissen, "The least-squares ambiguity decorrelation adjustment: a method for fast GPS integer ambiguity estimation," J. Geod., vol. 70, no. 1-2, pp. 65-82, Nov. 1995.

[12] P. Buist, "The Baseline Constrained LAMBDA Method for Single Epoch, Single Frequency Attitude Determination Applications," in 20th International Technical Meeting of The Satellite Division of the Institute of Navigation, Fort Worth, Texas, 2007.

[13] L. Baroni and H. K. Kuga, “Analysis of Attitude Determination Methods Using GPS Carrier Phase Measurements,” Math. Probl. Eng., vol. 2012, pp. 1-10, 2012.

[14] P. Teunissen and S. Verhagen, "On the Foundation of the Popular Ratio Test for GNSS Ambiguity Resolution," in 17th International Technical Meeting of The Satellite Division of the Institute of Navigation, Long Beach, California, 2004. 